Article

\title{
Deciphering S-RNase Allele Patterns in Cultivated and Wild Accessions of Italian Pear Germplasm
}

\author{
Stefania Bennici ${ }^{1,+}$, Mario Di Guardo ${ }^{1,+}{ }^{+}$, Gaetano Distefano ${ }^{1, *}{ }^{\mathbb{C}}$, Giuseppina Las Casas ${ }^{2}$, \\ Filippo Ferlito $^{2} \mathbb{D}$, Paolo De Franceschi ${ }^{3} \mathbb{D}$, Luca Dondini ${ }^{3}$, Alessandra Gentile ${ }^{1,4}$ and \\ Stefano La Malfa ${ }^{1}$ iD \\ 1 Dipartimento di Agricoltura, Alimentazione ed Ambiente, Università di Catania, Via Valdisavoia 5, \\ 95123 Catania, Italy; stefania.bennici@hotmail.it (S.B.); mario.diguardo@unict.it (M.D.G.); \\ gentilea@unict.it (A.G.); slamalfa@unict.it (S.L.M.) \\ 2 Consiglio per la ricerca in agricoltura e l'analisi dell'economia agraria, Centro di ricerca Olivicoltura, \\ Frutticoltura e Agrumicoltura, Corso Savoia, 190-95024 Acireale (CT), Italy; \\ giuseppina.lascasas@outlook.it (G.L.C.); filippo.ferlito@crea.gov.it (F.F.) \\ 3 Dipartimento di Scienze e Tecnologie Agroalimentari (DISTAL), Università degli Studi di Bologna, \\ 40126 Bologna, Italy; paolodefranceschi@gmail.com (P.D.F.); luca.dondini@unibo.it (L.D.) \\ 4 College of Horticulture and Landscape, Hunan Agricultural University, Changsha 410128, China \\ * Correspondence: distefag@unict.it \\ + Both authors contributed equally to this work.
}

Received: 4 November 2020; Accepted: 19 November 2020; Published: 22 November 2020

\begin{abstract}
The genus Pyrus is characterized by an S-RNase-based gametophytic self-incompatibility (GSI) system, a mechanism that promotes outbreeding and prevents self-fertilization. While the $S$-genotype of the most widely known pear cultivars was already described, little is known on the $S$-allele variability within local accessions. The study was conducted on 86 accessions encompassing most of the local Sicilian varieties selected for their traits of agronomic interest and complemented with some accessions of related wild species (P. pyrifolia Nakai, P. amygdaliformis Vill.) and some national and international cultivars used as references. The employment of consensus and specific primers enabled the detection of $24 \mathrm{~S}$-alleles combined in $48 \mathrm{~S}$-genotypes. Results shed light on the distribution of the $S$-alleles among accessions, with wild species and international cultivars characterized by a high diversity and local accessions showing a more heterogeneous distribution of the $S$-alleles, likely reflecting a more complex history of hybridization. The $S$-allele distribution was largely in agreement with the genetic structure of the studied collection. In particular, the "wild" genetic background was often characterized by the same S-alleles detected in P. pyrifolia and P. amygdaliformis. The analysis of the $S$-allele distribution provided novel insight into the contribution of the wild and international cultivars to the genetic background of the local Sicilian or national accessions. Furthermore, these results provide information that can be readily employed by breeders for the set-up of novel mating schemes.
\end{abstract}

Keywords: S-genotyping; S-locus; P. communis; P. pyrifolia; P. amygdaliformis; genetic structure

\section{Introduction}

European pear (Pyrus communis L.) is an economically important fruit tree species belonging to the Rosaceae family. Like the majority of the Rosaceae, the genus Pyrus exhibits the S-RNase-based gametophytic self-incompatibility (GSI) system, evolved by flowering plants to prevent self-fertilization and promote outbreeding [1]. The GSI system prevents self-fertilization through a specific pollen-pistil recognition that selectively inhibits the growth of those pollen tubes recognized by the pistil as "self" (i.e., pollen from the same plant or from individuals that are genetically related) [2]. The GSI system 
is controlled by the single, multigenic, and multiallelic $S$-locus expressing a female determinant in the style, the stylar ribonuclease (S-RNase) [3], and a male determinant in the pollen, a pool of F-box proteins known as SFBB (S-locus F-box brothers) [4]. In the GSI system, a match between the male $S$-determinant carried by the haploid genome of the pollen grain and one of the two $S$-alleles carried by the diploid genome of the stylar tissue (self-recognition) results in the arrest of the pollen tube growth triggered by the "self" S-RNase, which acts as a cytotoxin and possibly activates programmed cell death. Conversely, in non-self-recognition, S-RNase is inactivated by a specific F-box protein, through a proteolytic degradation mechanism [5]. Self-incompatibility is generally considered an undesired trait, especially for those cultivated species in which the success of the fertilization process is essential for fruit set. An understanding of the $S$-genotype is crucial for the choice of pollinators during the set-up of novel orchards and for novel breeding programs. Traditionally, the degree of compatibility between cultivars was determined directly in the field via the set-up of controlled crosses; however, this approach is time-consuming and often not reliable in the discrimination between fully and semi-compatible combinations. The advent of molecular biology techniques enabled both the sequencing of the gene coding for the S-RNase and the development of molecular markers, allowing a fast and relatively inexpensive screening of the $S$-genotypes in germplasms of interest [6]. The $S$-RNase gene is composed of five consensus conserved regions (C1, C2, C3, RC4, and C5) and the highly conserved noncanonical hexapeptide (IIWPNW); moreover, between C2 and C3 is located the hypervariable region (RHV) harboring a highly polymorphic intron. This RHV region has been largely exploited to assess the $S$-locus diversity in European pear by cloning and sequencing PCR products amplified with universal primers on the basis of conserved regions [6-16]. Sanzol [14] developed a PCR-based method for the detection of 20 S-RNase alleles in European pear by using consensus primers simultaneously amplifying a large number of alleles characterized by different intron sizes, plus a set of allele-specific primers. Then, further primer pairs were developed by Nikzad Gharehaghaji and colleagues [16], allowing the detection of additional alleles of European pear, some of which were highly similar and possibly derived from other Pyrus species.

Sicily is characterized by a wide pear biodiversity; to this extent, Mount Etna represents an ideal reservoir of different local accessions due to the occurrence of different microclimates, soils, and orographic conditions combined with the ancient history of cultivation and natural seed-based propagation. Moreover, the geographic location of Sicily in the Mediterranean Sea and its historical involvement in commercial exchanges may have favored the introgression of several traits of agronomical interest from different pear species. This wide pear biodiversity includes autochthonous and wild pears such as P. amygdaliformis Vill. and P. pyraster (L.) Burgsd. (P. communis ssp. pyraster L.) that were largely employed as rootstocks to increase the hardiness and longevity of the trees in past centuries [17]. P. amygdaliformis is native to the Mediterranean region and is highly tolerant to drought stress [18]. P. pyraster comes from the western Black Sea region, with a distribution area spanning from the British Isles to Latvia, and it is believed to be one of the most probable ancestors that gave rise to European pear $[19,20]$.

A previous study of genetic structure, conducted largely on the same accessions of the present study, revealed the presence of two subpopulations that can be reconducted to a "wild" and "cultivated" genetic status. Within the Sicilian local germplasm, only a small number of accessions were characterized by a high admixture of the two subpopulations, while the majority were characterized by a clear prevalence of one of the two subpopulations [17]. In such a genetic background, identifying the S-allele distribution within the subpopulations can provide valuable insights into the relationship and gene flow between wild and cultivated Sicilian pears.

In the present work, the PCR-based S-genotyping method described by Sanzol [14] was used to (i) ascertain the $S$-RNase composition in local Sicilian pear varieties and native wild accessions collected from the Mount Etna area in comparison with national and international varieties used as a reference, (ii) determine the distribution pattern of the $S$-alleles among the different groups 
of accessions, and (iii) compare the S-allele distribution in genotypes characterized by "wild" and "cultivated" genetic backgrounds.

\section{Materials and Methods}

\subsection{Plant Material and DNA Extraction}

The germplasm in analysis consisted of 86 accessions composed of 43 local varieties (LV), 16 individuals belonging to wild related species (RS) (nine P. pyraster and seven P. amydgaliformis, all collected from Mount Etna area, Italy), 18 nationally cultivated varieties (NCV), and nine internationally cultivated varieties (ICV) (Table 1). Accessions were located in two ex situ germplasm collections located in Catania district (Sicily, South Italy): the experimental farm of Catania University (UNICT, $10 \mathrm{~m}$ above sea level (a.s.l.)) and the Germplasm Bank of "Parco dell'Etna" (Mt. Etna, $850 \mathrm{~m}$ a.s.l.).

Genomic DNA was extracted from fresh leaves using ISOLATE II Plant DNA Kit (Bioline, Meridian Life Science, Memphis, TN, USA) following the protocol provided by the manufacturer. The concentration and the purity of the extracted DNA were assessed using a Nanodrop 2000 spectrophotometer (Thermo Scientific, Waltham, MA, USA) and agarose gel electrophoresis.

\subsection{S-Genotyping Assay}

The S-genotyping assay was performed according to the protocol described by Sanzol [14] using a pair of consensus primers, PycomC1F and PycomC5R [12], and 17 specific primers able to discriminate among 23 different S-RNase alleles (Table S1, Supplementary Materials). The S-genotyping assay was implemented with additional allele-specific primer pairs for alleles $\mathrm{PcS}_{126}$ and $\mathrm{PcS}_{127}$ (Table S1, Supplementary Materials). Primer pairs were ad hoc developed in this study on the basis of genomic sequences of $\mathrm{PcS}_{126}$ (accession number KF588567) and $\mathrm{PcS}_{127}$ (accession number KF588568) using Primer-BLAST [21]. The nine ICVs and the NCVs "Bella di Giugno", "Coscia", and "Gentile", which have a known S-genotype, were used as controls (Table S2, Supplementary Materials).

Consensus PCR was performed in a $20 \mu \mathrm{L}$ volume containing $40 \mathrm{ng}$ of genomic DNA, $1 \times$ PCR buffer II, $2 \mathrm{mM}$ magnesium chloride, $0.2 \mathrm{mM}$ dNTPs, $0.6 \mu \mathrm{M}$ each primer (PycomC1F and PycomC5R), and $1 \mathrm{U}$ of MyTaq DNA polymerase (Bioline, Meridian Life Science, Memphis, TN, USA). Amplification was conducted using a program with an initial denaturation at $94^{\circ} \mathrm{C}$ for $10 \mathrm{~min}$, followed by 35 cycles at $94{ }^{\circ} \mathrm{C}$ for $30 \mathrm{~s}, 57^{\circ} \mathrm{C}$ for $45 \mathrm{~s}$, and $72{ }^{\circ} \mathrm{C}$ for $2 \mathrm{~min}$, with a final cycle of $72{ }^{\circ} \mathrm{C}$ for $7 \mathrm{~min}$.

Allele-specific PCR was performed in a $20 \mu \mathrm{L}$ volume containing $40 \mathrm{ng}$ of genomic DNA, $1 \times$ PCR buffer II, $2 \mathrm{mM}$ magnesium chloride, $0.2 \mathrm{mM}$ dNTPs, from 0.3 to $0.6 \mu \mathrm{M}$ each primer (Table S1, Supplementary Materials), and $1 \mathrm{U}$ of MyTaq DNA polymerase (Bioline, Meridian Life Science, Memphis, TN, USA). Amplification was conducted using a program with an initial denaturation at $94{ }^{\circ} \mathrm{C}$ for $10 \mathrm{~min}$, followed by 35 cycles at $94{ }^{\circ} \mathrm{C}$ for $30 \mathrm{~s}, 58^{\circ} \mathrm{C}$ for $45 \mathrm{~s}$, and $72{ }^{\circ} \mathrm{C}$ for $1 \mathrm{~min}$, with a final cycle of $72{ }^{\circ} \mathrm{C}$ for $7 \mathrm{~min}$.

Amplicons were separated by gel electrophoresis in $1 \%$ agarose stained with SYBR Safe DNA gel stain (Invitrogen, Carlsbad, CA, USA). Image acquisition and fragment size estimation were performed using Image LabTM software with the GelDOCTM XR+ system (BIO-RAD Molecular Imager ${ }^{\circledR}$, Hercules, CA, USA).

\subsection{DNA Sequencing and Allele Identification}

Consensus PCR products were excised from agarose gel and purified using UPGRADE TO ISOLATE II Nucleic Acid Isolation Kits (Bioline, Meridian Life Science, Memphis, TN, USA) following the protocol provided by the manufacturer. Purified products were sequenced in the forward and reverse directions starting from primers PycomC1F1 and PycomC5R1 sequenced using an ABI310 genetic analyzer (Applied Biosystems, Foster City, CA, USA). 
Table 1. S-Genotypes and features of accessions employed in this study.

\begin{tabular}{|c|c|c|c|c|c|c|c|c|}
\hline Species & Accession Name & Origin & Status $^{a}$ & Collection $^{b}$ & Q1 & Q2 & Subpopulation $^{c}$ & $S$-Genotype \\
\hline Pyrus communis & Adamo & Italy (Sicily) & $\mathrm{LV}$ & UNICT & 0.053 & 0.947 & Wild & $\mathrm{PcS}_{105} / \mathrm{PcS}_{126}$ \\
\hline P. communis & Alessio & Italy (Sicily) & $\mathrm{LV}$ & Mt Etna & 0.97 & 0.03 & Cultivated & $\mathrm{PcS}_{107} /-$ \\
\hline P. communis & Angelico & Italy (Sicily) & $\mathrm{NCV}$ & Mt Etna & 0.989 & 0.011 & Cultivated & $\mathrm{PcS}_{104} / \mathrm{PcS}_{103}$ \\
\hline P. communis & Azzone di Cassone & Italy (Sicily) & LV & UNICT & 0.972 & 0.028 & Cultivated & $\mathrm{PcS}_{103} / \mathrm{PcS}_{127}$ \\
\hline P. communis & Bella di Giugno & Italy (Sicily) & $\mathrm{NCV}$ & Mt Etna & 0.054 & 0.946 & Wild & $\mathrm{PcS}_{104} / \mathrm{PcS}_{120}$ * \\
\hline P. communis & Bergamotto & Italy (Sicily) & $\mathrm{NCV}$ & UNICT & 0.984 & 0.016 & Cultivated & $\mathrm{PcS}_{103} / \mathrm{PcS}_{111}$ \\
\hline P. communis & Bianchetto & Italy (Sicily) & $\mathrm{NCV}$ & UNICT & 0.012 & 0.988 & Wild & $\mathrm{PcS}_{109} / \mathrm{PcS}_{117}$ \\
\hline P. communis & Bianchettone & Italy (Sicily) & LV & Mt Etna & 0.032 & 0.968 & Wild & $\mathrm{PcS}_{104} / \mathrm{PcS}_{105}$ \\
\hline P. communis & Bruttu Beddu & Italy (Sicily) & $\mathrm{LV}$ & Mt Etna & 0.929 & 0.071 & Cultivated & $\mathrm{PcS}_{103} /-$ \\
\hline P. communis & Buona Luisa & Italy (Sicily) & $\mathrm{NCV}$ & Mt Etna & 0.993 & 0.007 & Cultivated & $\mathrm{PcS}_{101} / \mathrm{PcS}_{103}$ \\
\hline P. communis & Butirra & Italy (Sicily) & $\mathrm{NCV}$ & UNICT & 0.991 & 0.009 & Cultivated & $\mathrm{PcS}_{101} / \mathrm{PcS}_{103}$ \\
\hline P. communis & Campana & Italy (Sicily) & $\mathrm{NCV}$ & UNICT & & & NA & $\mathrm{PcS}_{103} / \mathrm{PcS}_{122}$ \\
\hline P. communis & Catanese & Italy (Sicily) & LV & Mt Etna & 0.019 & 0.981 & Wild & $\mathrm{PcS}_{104} / \mathrm{PcS}_{108}$ \\
\hline P. communis & Cavaliere & Italy (Sicily) & $\mathrm{LV}$ & UNICT & & & NA & $\mathrm{PcS}_{104} / \mathrm{PcS}_{115}$ \\
\hline P. communis & Chiuzzu & Italy (Sicily) & $\mathrm{LV}$ & UNICT & 0.215 & 0.785 & Admixed & $\mathrm{PcS}_{103} / \mathrm{PcS}_{127}$ \\
\hline P. communis & Coscia & Italy (Sicily) & $\mathrm{NCV}$ & UNICT & 0.99 & 0.01 & Cultivated & $\mathrm{PcS}_{104} / \mathrm{PcS}_{103}$ * \\
\hline P. communis & Duchessa d'Angio' & Italy (Sicily) & LV & Mt Etna & 0.98 & 0.02 & Cultivated & $\mathrm{PcS}_{103} / \mathrm{PcS}_{105}$ \\
\hline P. communis & Faccia Donna & Italy (Sicily) & $\mathrm{LV}$ & UNICT & 0.009 & 0.991 & Wild & $\mathrm{PcS}_{124} /-$ \\
\hline P. communis & Faccibedda & Italy (Sicily) & $\mathrm{LV}$ & UNICT & 0.018 & 0.982 & Wild & $\mathrm{PcS}_{126} / \mathrm{PcS}_{127}$ \\
\hline P. communis & Franconello & Italy (Sicily) & $\mathrm{LV}$ & Mt Etna & & & NA & $\mathrm{PcS}_{126} / \mathrm{PcS}_{127}$ \\
\hline P. communis & Garibaldi & Italy (Sicily) & $\mathrm{LV}$ & Mt Etna & & & NA & $\mathrm{PcS}_{104} / \mathrm{PcS}_{115}$ \\
\hline P. communis & Garofalo & Italy (Sicily) & $\mathrm{NCV}$ & Mt Etna & 0.009 & 0.991 & Wild & $\mathrm{PCS}_{124} /-$ \\
\hline P. communis & Gentile & Italy (Sicily) & $\mathrm{NCV}$ & UNICT & 0.644 & 0.356 & Admixed & $\mathrm{PcS}_{101} / \mathrm{PcS}_{106}$ * \\
\hline P. communis & Ialufaru & Italy (Sicily) & $\mathrm{LV}$ & Mt Etna & 0.014 & 0.986 & Wild & $\mathrm{PcS}_{124} /-$ \\
\hline P. communis & Ianculiddu & Italy (Sicily) & $\mathrm{LV}$ & Mt Etna & 0.012 & 0.988 & Wild & $\mathrm{PcS}_{125} / \mathrm{PcS}_{127}$ \\
\hline P. communis & Iazzuleddu & Italy (Sicily) & $\mathrm{LV}$ & UNICT & 0.984 & 0.016 & Cultivated & $\mathrm{PcS}_{103} / \mathrm{PcS}_{127}$ \\
\hline P. communis & Mezza Campana & Italy (Sicily) & $\mathrm{LV}$ & Mt Etna & & & NA & $\mathrm{PcS}_{104} / \mathrm{PcS}_{108}$ \\
\hline P. communis & Moscatello 1 & Italy (Sicily) & $\mathrm{NCV}$ & Mt Etna & 0.018 & 0.982 & Wild & $\mathrm{PcS}_{104} / \mathrm{PcS}_{123}$ \\
\hline P. communis & Moscatello 2 & Italy (Sicily) & $\mathrm{NCV}$ & UNICT & 0.027 & 0.973 & Wild & $\mathrm{PcS}_{103} / \mathrm{PcS}_{116}$ \\
\hline P. communis & Moscatello Maiolino & Italy (Sicily) & $\mathrm{LV}$ & Mt Etna & & & NA & $\mathrm{PcS}_{126} / \mathrm{PcS}_{127}$ \\
\hline P. communis & Moscatello Nero & Italy (Sicily) & $\mathrm{LV}$ & Mt Etna & 0.014 & 0.986 & Cultivated & $\mathrm{PcS}_{101} / \mathrm{PcS}_{106}$ \\
\hline P. communis & Paradiso/Confittaru & Italy (Sicily) & $\mathrm{LV}$ & Mt Etna & 0.081 & 0.919 & Wild & $\mathrm{PcS}_{103} / \mathrm{PcS}_{126}$ \\
\hline P. communis & Pasqualino & Italy (Sicily) & $\mathrm{LV}$ & UNICT & 0.968 & 0.032 & Cultivated & $\mathrm{PcS}_{103} / \mathrm{PcS}_{111}$ \\
\hline P. communis & Pauluzzo & Italy (Sicily) & $\mathrm{LV}$ & Mt Etna & 0.018 & 0.982 & Wild & $\mathrm{PcS}_{126} / \mathrm{PcS}_{127}$ \\
\hline P. communis & Pergolesi & Italy (Sicily) & LV & Mt Etna & 0.989 & 0.011 & Cultivated & $\mathrm{PcS}_{101} / \mathrm{PcS}_{103}$ \\
\hline
\end{tabular}


Table 1. Cont.

\begin{tabular}{|c|c|c|c|c|c|c|c|c|}
\hline Species & Accession Name & Origin & Status ${ }^{a}$ & Collection $^{b}$ & Q1 & Q2 & Subpopulation ${ }^{c}$ & S-Genotype \\
\hline P. communis & Piccola Dolce & Italy (Sicily) & $\mathrm{LV}$ & Mt Etna & & & NA & $\mathrm{PcS}_{104} / \mathrm{PcS}_{117}$ \\
\hline P. communis & Piridda & Italy (Sicily) & $\mathrm{LV}$ & Mt Etna & 0.007 & 0.993 & Wild & $\mathrm{PcS}_{109} / \mathrm{PcS}_{117}$ \\
\hline P. communis & Piru Mulinciana & Italy (Sicily) & $\mathrm{LV}$ & Mt Etna & 0.99 & 0.01 & Cultivated & $\mathrm{PcS}_{101} / \mathrm{PcS}_{108}$ \\
\hline P. communis & Piru Pizzu & Italy (Sicily) & $\mathrm{LV}$ & Mt Etna & 0.139 & 0.861 & Wild & $\mathrm{PcS}_{105} / \mathrm{PcS}_{127}$ \\
\hline P. communis & Pisciazzanu & Italy (Sicily) & $\mathrm{LV}$ & Mt Etna & 0.056 & 0.944 & Wild & $\mathrm{PcS}_{101} /-$ \\
\hline P. communis & Pistacchino & Italy (Sicily) & $\mathrm{LV}$ & Mt Etna & 0.699 & 0.301 & Admixed & $\mathrm{PcS}_{117} / \mathrm{PcS}_{121}$ \\
\hline P. communis & Putiru d'Estate & Italy (Sicily) & $\mathrm{LV}$ & Mt Etna & 0.985 & 0.015 & Cultivated & $\mathrm{PcS}_{101} / \mathrm{PcS}_{103}$ \\
\hline P. communis & Putiru d'Inverno & Italy (Sicily) & $\mathrm{LV}$ & UNICT & 0.992 & 0.008 & Cultivated & $\mathrm{PcS}_{103} /-$ \\
\hline P. communis & Razzuolo Rosata & Italy (Sicily) & $\mathrm{LV}$ & Mt Etna & 0.017 & 0.983 & Wild & $\mathrm{PcS}_{103} / \mathrm{PcS}_{106}$ \\
\hline P. communis & Regina & Italy (Sicily) & LV & UNICT & & & NA & $\mathrm{PcS}_{101} / \mathrm{PcS}_{122}$ \\
\hline P. communis & Rosa & Italy (Sicily) & $\mathrm{LV}$ & Mt Etna & 0.01 & 0.99 & Wild & $\mathrm{PcS}_{104} / \mathrm{PcS}_{103}$ \\
\hline P. communis & San Cono & Italy (Sicily) & $\mathrm{LV}$ & Mt Etna & 0.651 & 0.349 & Admixed & $\mathrm{PcS}_{102} / \mathrm{PcS}_{103}$ \\
\hline P. communis & San Giovanni & Italy (Sicily) & $\mathrm{NCV}$ & UNICT & 0.009 & 0.991 & Wild & $\mathrm{PcS}_{102} / \mathrm{PcS}_{109}$ \\
\hline P. communis & San Giovannino & Italy (Sicily) & $\mathrm{LV}$ & Mt Etna & 0.01 & 0.99 & Wild & $\mathrm{PcS}_{105} /-$ \\
\hline P. communis & San Pietro & Italy (Sicily) & $\mathrm{NCV}$ & UNICT & 0.015 & 0.985 & Wild & $\mathrm{PcS}_{120} / \mathrm{PcS}_{126}$ \\
\hline P. communis & Santa Caterina & Italy (Sicily) & LV & UNICT & 0.01 & 0.99 & Wild & $\mathrm{PcS}_{104} / \mathrm{PcS}_{125}$ \\
\hline P. communis & Sciaduna & Italy (Sicily) & $\mathrm{LV}$ & Mt Etna & 0.05 & 0.95 & Wild & $\mathrm{PcS}_{111} / \mathrm{PcS}_{117}$ \\
\hline P. communis & Spadona & Italy (Sicily) & $\mathrm{NCV}$ & Mt Etna & 0.029 & 0.971 & Wild & $\mathrm{PcS}_{104} / \mathrm{PcS}_{105}$ \\
\hline P. communis & Spineddu & Italy (Sicily) & $\mathrm{NCV}$ & UNICT & 0.039 & 0.961 & Wild & $\mathrm{PcS}_{111} / \mathrm{PcS}_{117}$ \\
\hline P. communis & Tabaccaro & Italy (Sicily) & LV & Mt Etna & 0.029 & 0.971 & Wild & $\mathrm{PcS}_{101} / \mathrm{PcS}_{127}$ \\
\hline P. communis & Ucciarduni & Italy (Sicily) & $\mathrm{NCV}$ & UNICT & & & NA & $\mathrm{PcS}_{103} / \mathrm{PcS}_{108}$ \\
\hline P. communis & Urzi' & Italy (Sicily) & LV & UNICT & & & NA & $\mathrm{PcS}_{103} / \mathrm{PcS}_{125}$ \\
\hline P. communis & Villalba & Italy (Sicily) & $\mathrm{LV}$ & Mt Etna & 0.276 & 0.724 & Admixed & $\mathrm{PcS}_{101} / \mathrm{PcS}_{109}$ \\
\hline P. communis & Virgolese & Italy (Sicily) & $\mathrm{NCV}$ & UNICT & 0.99 & 0.01 & Cultivated & $\mathrm{PcS}_{101} / \mathrm{PcS}_{103}$ \\
\hline P. communis & Zio Pietro & Italy (Sicily) & $\mathrm{LV}$ & UNICT & 0.986 & 0.014 & Cultivated & $\mathrm{PcS}_{101} / \mathrm{PcS}_{108}$ \\
\hline P. communis & Zuccareddu & Italy (Sicily) & $\mathrm{LV}$ & Mt Etna & 0.034 & 0.966 & Wild & $\mathrm{PcS}_{101} / \mathrm{PcS}_{107}$ \\
\hline P. amygdaliformis & 1 & Italy (Sicily) & RS & $\begin{array}{c}\text { North (N) } 37^{\circ} 47^{\prime} 692^{\prime \prime} \text { east } \\
\text { (E) } 14^{\circ} 50^{\prime} 925^{\prime \prime}\end{array}$ & 0.013 & 0.987 & Wild & $\mathrm{PcS}_{120} / \mathrm{PcS}_{122}$ \\
\hline P. amygdaliformis & 2 & Italy (Sicily) & RS & N $37^{\circ} 51^{\prime} 189^{\prime \prime}$ E $14^{\circ} 50^{\prime} 795^{\prime \prime}$ & 0.008 & 0.992 & Wild & $\mathrm{PcS}_{126} /-$ \\
\hline P. amygdaliformis & 3 & Italy (Sicily) & RS & N $37^{\circ} 51^{\prime} 147^{\prime \prime}$ E $14^{\circ} 50^{\prime} 739^{\prime \prime}$ & 0.007 & 0.993 & Wild & $\mathrm{PcS}_{101} / \mathrm{PcS}_{116}$ \\
\hline P. amygdaliformis & 4 & Italy (Sicily) & RS & $\mathrm{N} 37^{\circ} 51^{\prime} 109^{\prime \prime} \mathrm{E} 14^{\circ} 50^{\prime} 809^{\prime \prime}$ & 0.007 & 0.993 & Wild & $\mathrm{PcS}_{116} / \mathrm{PcS}_{125}$ \\
\hline P. amygdaliformis & 5 & Italy (Sicily) & RS & N $37^{\circ} 51^{\prime} 116^{\prime \prime}$ E $14^{\circ} 50^{\prime} 806^{\prime \prime}$ & & & NA & $\mathrm{PcS}_{120} / \mathrm{PcS}_{122}$ \\
\hline P. amygdaliformis & 7 & Italy (Sicily) & RS & N $37^{\circ} 51^{\prime} 139^{\prime \prime}$ E $14^{\circ} 50^{\prime} 765^{\prime \prime}$ & 0.009 & 0.991 & Wild & $\mathrm{PcS}_{108} / \mathrm{PcS}_{125}$ \\
\hline P. amygdaliformis & 10 & Italy (Sicily) & RS & N $37^{\circ} 51^{\prime} 137^{\prime \prime}$ E $14^{\circ} 50^{\prime} 748^{\prime \prime}$ & & & NA & $\mathrm{PcS}_{106} /-$ \\
\hline
\end{tabular}


Table 1. Cont

\begin{tabular}{|c|c|c|c|c|c|c|c|c|}
\hline Species & Accession Name & Origin & Status $^{a}$ & Collection ${ }^{b}$ & Q1 & Q2 & Subpopulation $^{\mathrm{c}}$ & S-Genotype \\
\hline P. pyraster & 2 & Italy (Sicily) & RS & $\mathrm{N} 37^{\circ} 48^{\prime} 143^{\prime \prime}$ E $14^{\circ} 51^{\prime} 203^{\prime \prime}$ & & & NA & $\mathrm{PcS}_{111 /-}$ \\
\hline P. pyraster & 3 & Italy (Sicily) & RS & N $37^{\circ} 51^{\prime} 796^{\prime \prime}$ E $14^{\circ} 52^{\prime} 409^{\prime \prime}$ & 0.036 & 0.964 & Wild & $\mathrm{PcS}_{122} /-$ \\
\hline P. pyraster & 4 & Italy (Sicily) & RS & $\mathrm{N} 37^{\circ} 52^{\prime} 808^{\prime \prime} \mathrm{E} 14^{\circ} 52^{\prime} 571^{\prime \prime}$ & & & NA & $\mathrm{PcS}_{127} /-$ \\
\hline P. pyraster & 5 & Italy (Sicily) & RS & $\mathrm{N} 37^{\circ} 52^{\prime} 868^{\prime \prime} \mathrm{E} 14^{\circ} 52^{\prime} 565^{\prime \prime}$ & & & NA & $\mathrm{PcS}_{105} / \mathrm{PcS}_{123}$ \\
\hline P. pyraster & 7 & Italy (Sicily) & RS & N $37^{\circ} 47^{\prime} 691^{\prime \prime}$ E $14^{\circ} 50^{\prime} 901^{\prime \prime}$ & 0.097 & 0.903 & Wild & $\mathrm{PcS}_{101} / \mathrm{PcS}_{111}$ \\
\hline P. pyraster & 8 & Italy (Sicily) & RS & N $37^{\circ} 52^{\prime} 006^{\prime \prime}$ E $14^{\circ} 52^{\prime} 969^{\prime \prime}$ & 0.007 & 0.993 & Wild & $\mathrm{PcS}_{108} / \mathrm{PcS}_{127}$ \\
\hline P. pyraster & 9 & Italy (Sicily) & RS & N $37^{\circ} 51^{\prime} 865^{\prime \prime}$ E $14^{\circ} 52^{\prime} 672^{\prime \prime}$ & 0.009 & 0.991 & Wild & $\mathrm{PcS}_{105} / \mathrm{PcS}_{109}$ \\
\hline P. pyraster & 10 & Italy (Sicily) & $\mathrm{RS}$ & $\mathrm{N} 37^{\circ} 48^{\prime} 276^{\prime \prime} \mathrm{E} 14^{\circ} 51^{\prime} 269^{\prime \prime}$ & 0.477 & 0.523 & Admixed & $\mathrm{PcS}_{104} / \mathrm{PcS}_{110}$ \\
\hline P. pyraster & 11 & Italy (Sicily) & RS & N $37^{\circ} 53^{\prime} 038^{\prime \prime}$ E $14^{\circ} 52^{\prime} 578^{\prime \prime}$ & 0.057 & 0.943 & Wild & $\mathrm{PcS}_{108} /-$ \\
\hline P. communis & Abbé Fétel & France & ICV & UNIBO & 0.967 & 0.033 & Cultivated & $\mathrm{PcS}_{104} / \mathrm{PcS}_{105}$ * \\
\hline P. communis & Beurre Hardy & USA & $\mathrm{ICV}$ & UNIBO & 0.391 & 0.609 & Admixed & $\mathrm{PcS}_{108} / \mathrm{PcS}_{114}$ * \\
\hline P. communis & Cascade & USA & $\mathrm{ICV}$ & UNIBO & & & NA & $\mathrm{PcS}_{101} / \mathrm{PcS}_{104}$ * \\
\hline P. communis & Dr. Jules Guyot & France & ICV & UNIBO & & & NA & $\mathrm{PcS}_{101} / \mathrm{PcS}_{105}$ * \\
\hline P. communis & Kaiser & France & ICV & UNIBO & 0.914 & 0.086 & Cultivated & $\mathrm{PcS}_{107} / \mathrm{PcS}_{125}$ * \\
\hline P. communis & Max Red Barlet & USA & $\mathrm{ICV}$ & UNIBO & 0.993 & 0.007 & Cultivated & $\mathrm{PcS}_{101} / \mathrm{PcS}_{102}$ * \\
\hline P. communis & Old Home & USA & ICV & UNIBO & & & NA & $\mathrm{PcS}_{101} / \mathrm{PcS}_{113}$ * \\
\hline P. communis & Harrow Sweet & Canada & ICV & UNIBO & 0.987 & 0.013 & Cultivated & $\mathrm{PcS}_{102} / \mathrm{PcS}_{105}$ * \\
\hline P. communis & Williams & England & ICV & UNIBO & 0.993 & 0.007 & Cultivated & $\mathrm{PcS}_{101} / \mathrm{PcS}_{102}$ * \\
\hline
\end{tabular}




\subsection{Clustering}

S-RNase alleles identified for each genotype were converted into a binary matrix and used to compute a principal component analysis (PCA) on the basis of a dissimilarity matrix, performed using the statistical package $\mathrm{R}$ [22].

\section{Results}

The germplasm was genotyped using the consensus primers PycomC1F1 and PycomC5R [12]. The analysis of the PCR products allowed the identification of six alleles: $\mathrm{PcS}_{101}(1300 \mathrm{bp}), \mathrm{PcS}_{102}$ (1700 bp), PcS 104 (750 bp), PcS 110 (2200 bp), PcS 113 (2000 bp), and PcS $\mathrm{P}_{120}$ (800 bp), while 16 S-alleles were identified through the use of specific primers (Table S1, Supplementary Materials). The consensus PCR products and the amplification for each $S$-RNase allele tested for every accession are shown in the Table S2 (Supplementary Materials).

The accession "Iazzuleddu" showed a consensus amplicon of $1650 \mathrm{bp}$, positive to $\mathrm{PcS}_{103}$ primers and a new PCR product size of approximately $850 \mathrm{bp}$, which could not be identified by any of the tested allele-specific primers. The same amplicon was detected in the LVs "Azzone di Cassone", "Chiuzzu", "Faccibedda", "Franconello", "Ianculiddu", "Moscatello maiolino", "Pauluzzo", "Piru Pizzu", and "Tabaccaro" and in the two RS genotypes of P. pyraster (no. 4 and no. 8). The sequencing of the 850 bp amplicon of "Iazzuleddu" showed a $100 \%$ similarity to the S-RNase-PcS 127 allele of Pyrus communis (Sequence ID: KF588568.1). A new pair of primers was designed to selectively amplify the S-RNase-PcS ${ }_{127}$ allele (Table S1, Supplementary Materials) and allowed its detection in all genotypes carrying the band of $850 \mathrm{bp}$ producing an amplicon of $214 \mathrm{bp}$.

The LV "Pauluzzo", in addition to the band of $850 \mathrm{bp}$ ( $\mathrm{PcS}_{127}$ allele), was characterized by a smaller band of $680 \mathrm{bp}$ that was not identified by any of the allele-specific primers. The sequencing of the $680 \mathrm{bp}$ amplicon revealed a $99 \%$ similarity to the S-RNase-PcS 126 allele of Pyrus communis (Sequence ID: KF588567.1). This amplicon was also detected in the LVs "Adamo", "Faccibedda", "Franconello", "Moscatello maiolino", and "Paradiso Confittaru", in the NCV "S. Pietro", and in the RS P. amygdaliformis (no. 2). A specific primer pair was designed to selectively amplify the S-RNase-PcS 126 allele (Table S1, Supplementary Materials) producing an amplicon of $100 \mathrm{bp}$ in all genotypes carrying the initial band of $680 \mathrm{bp}$.

Summing up, the use of the consensus, the specific, and the two ad hoc designed primers allowed the detection of $24 S$-alleles; for 72 accessions, both $S$-alleles were detected (resulting in 48 different $S$-genotypes), while, for the remaining 14 accessions, only a single allele was detected (Table 1 ). The relative frequencies of the $S$-RNase alleles identified for each group (ICV, NCV, LV, and RS) are shown in Table 2.

The S-allele showing the highest absolute frequency in the germplasm was $\mathrm{PcS}_{103}$, detected in 23 accessions (Table 2). Looking at the distribution of the $S$-RNase allele among the four pear groups, $\mathrm{PcS}_{103}$ was detected only among Italian varieties (14 LVs and nine NCVs) (Table 2). The S-RNase alleles $\mathrm{PcS}_{101}, \mathrm{PcS}_{104}, \mathrm{PcS}_{105}$, and $\mathrm{PcS}_{108}$ (identified in 21, 16, 11, and nine accessions, respectively) were detected, although with different frequencies, in all four groups; in contrast, five $S$-RNase alleles ( $\mathrm{PcS}_{110}, \mathrm{PcS}_{113}, \mathrm{PcS}_{114}, \mathrm{PcS}_{115}$, and $\left.\mathrm{PcS}_{121}\right)$ were group-specific $\left(\mathrm{PcS}_{110}\right.$ for RS, $\mathrm{PcS}_{113}$ and $\mathrm{PcS}_{114}$ for $\mathrm{ICV}$, and $\mathrm{PcS}_{115}$ and $\mathrm{PcS}_{121}$ for LV) (Table 2). None of the S-RNase alleles detected in more than two samples were found in only one of the four classes presented (Table 2).

A number of 68 out of the 86 accessions here characterized were previously SSR-genotyped, and genetic structure analysis detected two subpopulations defined as "wild" and "cultivated" [17] (Table 1). The "wild" subpopulation largely characterized the RS group (contributing for an average of 93.4\% on the genetic makeup of such accessions), while the "cultivated" subpopulation was predominantly detected in the ICVs (average of $87.4 \%$ ). A more complex pattern was detected for the accessions deemed as LVs or NCVs; in both cases most of the accessions showed a clear prevalence (more than the $80 \%$ ) of one of the two subpopulations with only five accessions showing a more balanced presence of the "wild" and "cultivated" subpopulations ("admixed" [17]). Figure 1 showed the relative frequency 
of the different $S$-alleles according to the structure analysis (subpopulations "wild", "cultivated", and "admixed").

Table 2. S-Allele frequencies among analyzed accessions.

\begin{tabular}{|c|c|c|c|c|c|}
\hline$S$-Allele & Count & RS & LV & $\mathrm{NCV}$ & ICV \\
\hline $\mathrm{PcS}_{101}$ & 21 & 0.1 & 0.48 & 0.19 & 0.24 \\
\hline $\mathrm{PcS}_{102}$ & 5 & 0 & 0.2 & 0.2 & 0.6 \\
\hline $\mathrm{PcS}_{103}$ & 23 & 0 & 0.61 & 0.39 & 0 \\
\hline $\mathrm{PcS}_{104}$ & 16 & 0.06 & 0.5 & 0.31 & 0.13 \\
\hline $\mathrm{PcS}_{105}$ & 11 & 0.18 & 0.45 & 0.09 & 0.27 \\
\hline $\mathrm{PcS}_{106}$ & 4 & 0.25 & 0.5 & 0.25 & 0 \\
\hline $\mathrm{PcS}_{107}$ & 3 & 0 & 0.67 & 0 & 0.33 \\
\hline $\mathrm{PcS}_{108}$ & 9 & 0.33 & 0.44 & 0.11 & 0.11 \\
\hline $\mathrm{PcS}_{109}$ & 5 & 0.2 & 0.4 & 0.4 & 0 \\
\hline $\mathrm{PcS}_{110}$ & 1 & 1 & 0 & 0 & 0 \\
\hline $\mathrm{PcS}_{111}$ & 6 & 0.33 & 0.33 & 0.33 & 0 \\
\hline $\mathrm{PcS}_{113}$ & 1 & 0 & 0 & 0 & 1 \\
\hline $\mathrm{PcS}_{114}$ & 1 & 0 & 0 & 0 & 1 \\
\hline $\mathrm{PcS}_{115}$ & 2 & 0 & 1 & 0 & 0 \\
\hline $\mathrm{PcS}_{116}$ & 3 & 0.67 & 0 & 0.33 & 0 \\
\hline $\mathrm{PcS}_{117}$ & 6 & 0 & 0.67 & 0.33 & 0 \\
\hline $\mathrm{PcS}_{120}$ & 4 & 0.5 & 0 & 0.5 & 0 \\
\hline $\mathrm{PcS}_{121}$ & 1 & 0 & 1 & 0 & 0 \\
\hline $\mathrm{PcS}_{122}$ & 5 & 0.6 & 0.2 & 0.2 & 0 \\
\hline $\mathrm{PcS}_{123}$ & 2 & 0.5 & 0 & 0.5 & 0 \\
\hline $\mathrm{PcS}_{124}$ & 3 & 0 & 0.67 & 0.33 & 0 \\
\hline $\mathrm{PcS}_{125}$ & 6 & 0.33 & 0.5 & 0 & 0.17 \\
\hline $\mathrm{PcS}_{126}$ & 8 & 0.13 & 0.75 & 0.13 & 0 \\
\hline $\mathrm{PcS}_{127}$ & 12 & 0.17 & 0.83 & 0 & 0 \\
\hline
\end{tabular}

For each of the $S$-alleles detected, the absolute frequency is reported together with the relative frequency according to the four classes: RS (wild related species), LV (local varieties), NCV (nationally cultivated varieties), and ICV (internationally cultivated varieties).

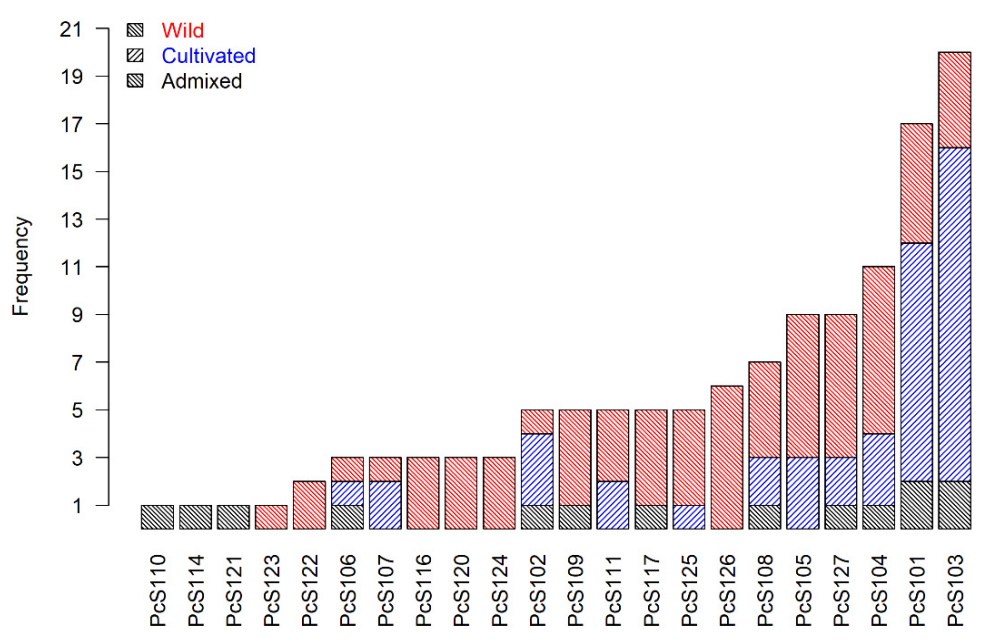

Figure 1. S-Allele frequency among analyzed accessions according to their population structure information shown in Bennici et al. (2018) [17]. Samples without an assigned subpopulation are excluded.

Results indicated that the two most abundant S-alleles, $\mathrm{PcS}_{103}$ and $\mathrm{PcS}_{101}$, were largely detected in accessions characterized by a clear predominance of the "cultivated" subpopulation $(60 \%$ and $56 \%$, respectively; blue color in Figure 1), whereas the other $S$-alleles were mostly associated with individuals characterized by the "wild" subpopulation (red color in Figure 1). The alleles $\mathrm{PcS}_{116}, \mathrm{PcS}_{120}, \mathrm{PcS}_{122}$, 
$\mathrm{PcS}_{123}, \mathrm{PcS}_{124}$, and $\mathrm{PcS}_{126}$ (18 accessions in total) were detected only in samples showing a "wild" genetic background (Figure 1).

The results presented in Table S2 (Supplementary Materials) were converted into a binary matrix and used to compute a PCA (Figure 2).

A

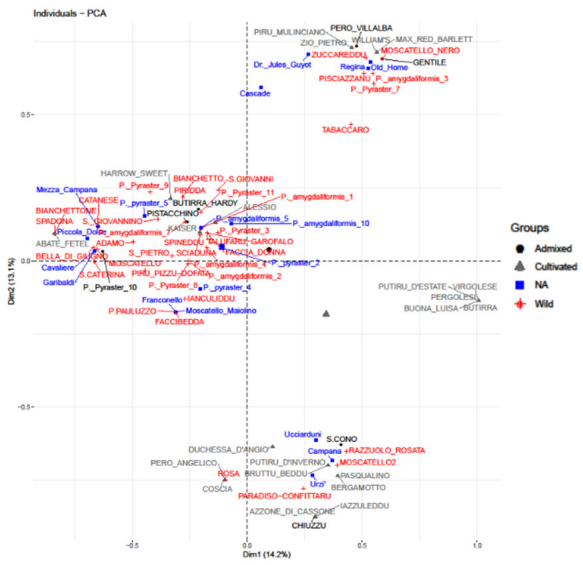

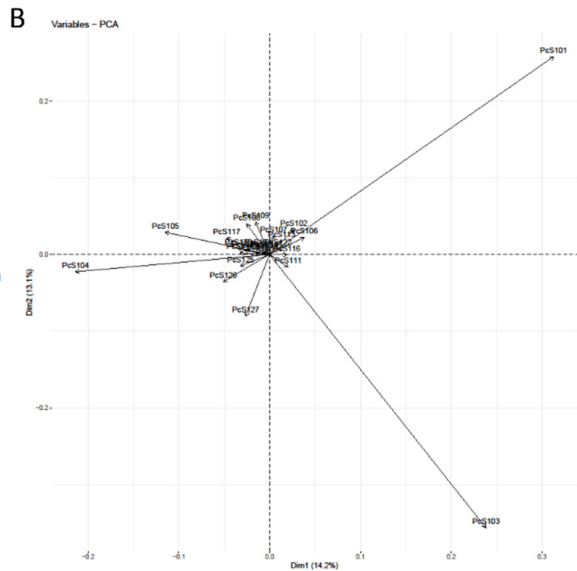

Figure 2. Principal component analysis (PCA) depicting the distribution of accessions over the first two PCs (Dim1 and Dim2) performed on the genetic data scored for the presence/absence of each S-allele. (A) PCA biplot: different colors indicate the subpopulations detected with structure analysis in Bennici et al. (2018) [17]: "wild" (red); "cultivated" (blue); "admixed" (black); not available (gray). (B) Loading projections of the variables employed.

The first two PCs explained $27.3 \%$ of the whole variability $(14.2 \%$ and $13.1 \%$ for PC1 and PC2, respectively). The analysis of the first two principal components (Dim1 and Dim2) allowed the definition of four clusters composed of five $\left(\operatorname{Dim} 1>0\right.$, Dim2 $\sim$, S-genotype $\left.=\mathrm{PcS}_{101}, \mathrm{PcS}_{103}\right)$, $16\left(\operatorname{Dim} 1>0\right.$, Dim2 > 0, one $\left.\mathrm{PcS}_{101}\right), 18\left(\operatorname{Dim} 1 \sim 0\right.$, Dim2 < 0, one $\left.\mathrm{PcS}_{103}\right)$, and 47 (Dim1 < 0, Dim2 0, other $S$-alleles) accessions. The five accessions characterized by a $\mathrm{PcS}_{101} / \mathrm{PcS}_{103} S$-genotype included the NCVs "Butirra", "Buona Luisa", and "Virgolese", and the LVs "Putiru d'Estate" and "Pergolesi" (Figure 2). Even though the PCA was computed with S-allele data, the first principal component (Dim1) was highly predictive for the genetic structure results, with individuals plotted in the upper-right and lower-right quadrants (Dim1 $>0$ ) showing a predominance of the "cultivated" subpopulation (blue color), while samples characterized by Dim1 negative values were largely "wild" (red color).

\section{Discussion}

Despite its importance for breeding and as an agronomic trait, information on GSI genetic background is mainly known for the most commonly used varieties. The germplasm collection herein analyzed encompassed local cultivars selected through the last two centuries for their traits of agronomical interest such as chilling requirements, resistance to biotic/abiotic stress, and fruit quality. The present work aimed to decipher the $S$-genotype of such local varieties and to assess similarity and differences with the close wild accessions P. pyraster and P. amygdaliformis and with some national and international cultivars of P. communis. Analyses were carried out employing the PCR-based S-genotyping method described by Sanzol [14], resulting in the identification of 24 S-alleles [14,16]. S-RNase alleles identified in the reference ICVs and the NCVs "Bella di Giugno", "Coscia", and "Gentile" agreed with previous reports $[6,8,10,11,14,23,24]$, confirming the reliability of the protocol for the detection of the known European pear S-RNases.

S-Allele genotyping allowed the definition of the complete $S$-genotype for $84 \%$ of the accessions, while, for the remaining 14 samples, only one allele was identified. Given the forced heterozygosity at the S-locus, the detection of a single amplicon (Table S2, Supplementary Materials) implied the presence of additional alleles that were undetected suggesting the occurrence of sequence diversity 
and/or of insertions/deletions (INDELs) at the probe site [25]. These 14 genotypes included mostly LVs and RSs, reinforcing the assumption that the gene pool of pears from the Etna region is, to a certain extent, different from that of the widely employed cultivars.

$S$-Alleles were not uniformly distributed across the germplasm; in particular, the most abundant $S$-alleles were $\mathrm{PcS}_{103}$, detected in 23 accessions (61\% LV; 39\% NCV), and $\mathrm{PcS}_{101}$ detected in 21 accessions (10\% RS; 48\% LV; 19\% NCV; 24\% ICV) (Table 2). Fourteen S-alleles were detected in five or fewer accessions each (Table 2). Such variability reflected the different history and utilization of the accessions, with NCV or ICV cultivars widely employed both for cultivation and in breeding plans, such as "Coscia", "Gentile", "Cascade", "Dr. Jules Guyot", "Max Red Bartlett", "Old home", and "Williams", contributing to the spread of either $\mathrm{PcS}_{103}$ or $\mathrm{PcS}_{101}$. Conversely, only two RS (P. amygdaliformis n.3 and P. pyraster n.7) showed $\mathrm{PcS}_{101}$ and none showed $\mathrm{PcS}_{103}$ (Table 1).

None of the most abundant $S$-alleles were exclusively detected in one of the four classes in which the germplasm was subdivided according to its diffusion, suggesting a high heterogeneity within each of the groups, with LV and NCV accessions being highly similar to either RS or ICV.

This result agreed with the findings of the structure analysis performed by Bennici et al. [17] in which the presence of two subpopulations ("wild" and "cultivated") was revealed and that, although characterizing the RSs and ICVs, respectively, coexisted in the LVs and NCVs. Within these two groups, accessions were either "wild" or "cultivated" with very few accessions showing an admixed genetic configuration. The high genetic diversity between RSs and ICVs was here confirmed by the analysis of the S-alleles; in fact, 10 S-alleles $\left(\mathrm{PcS}_{106}, \mathrm{PcS}_{109}, \mathrm{PcS}_{110}, \mathrm{PcS}_{111}, \mathrm{PcS}_{116}, \mathrm{PcS}_{120}, \mathrm{PcS}_{122}, \mathrm{PcS}_{123}, \mathrm{PcS}_{126}\right.$, and $\left.\mathrm{PcS}_{127}\right)$, detected in at least one RS accession, were absent in the ICVs, and four S-alleles $\left(\mathrm{PcS}_{102}\right.$, $\mathrm{PcS}_{107}, \mathrm{PcS}_{113}, \mathrm{PcS}_{114}$ ) were detected in ICVs and absent in RS accessions.

Interestingly, when the $S$-allele genotypic data were matched with the population structure results, the most frequent $S$-alleles, $\mathrm{PcS}_{103}$ and $\mathrm{PcS}_{101}$, were found largely present in the "cultivated" group (Figure 1), while the remaining S-alleles were exclusively or predominantly detected in the "wild" accessions. The PCA analysis confirmed the close relationship between $S$-alleles and the genetic stratification of the germplasm collection with accessions that largely clustered according to their "wild" or "cultivated" nature (Figure 2).

Among the S-alleles largely present in the "wild" subpopulation, $\mathrm{PcS}_{126}$ and $\mathrm{PcS}_{127}$, were detected in 20 accessions through the design of specific primers (Table 2), the same $S$-alleles were already detected among Iranian local varieties [16]. Nikzad Gharehaghaji and colleagues [16] highlighted the high sequence homology of $\mathrm{PcS}_{126}$ and $\mathrm{PcS}_{127}$ with the $S$-alleles of the Asian pear species $P$. korshinskyi (S9) and P. $\times$ bretschneideri (S19), respectively, suggesting that these alleles might have been introgressed by hybridization. In fact, P. korshinskyi Litv. is native to Central Asia, and it was proposed to have originated from the hybridization between $P$. communis and $P$. regelii Rehder., a species native of Afghanistan [26-28]. The Chinese white pear (.$\times$ bretschneideri Rehder.) is native of East Asia [28]. Molecular studies using RAPD and SSR markers suggested that $P . \times$ bretschneideri might share a common ancestor with P. pyrifolia [29,30]. Recently, a genotyping-by-sequencing (GBS) study proposed that the genomes of the Ussurian pear (P. ussuriensis Maxim.) and the Chinese sand pear (P. pyrifolia Nakai) could have both contributed to the origin of P. $\times$ bretschneideri [31]. Interestingly, four LVs ("Faccibedda", "Pauluzzo", "Moscatello Maiolino", and "Franconello") showed both alleles PCS127 and $\mathrm{PC}_{\mathrm{S} 126}$, suggesting a possible contribution of Asian pear species on the genetic backgrounds of these accessions.

The S-RNase allele PcS 117 (detected in the NCV "Bianchetto" and the LVs "Piccola Dolce", "Piridda", "Pistacchino", "Sciaduna", and "Spineddu", Table 1) was amplified using the primer pairs developed for the $\mathrm{PpS}_{9}$ allele of the Japanese pear P. pyrifolia. $\mathrm{PpS}_{9}$ is one of the most common $S$-alleles characterizing Japanese pear; nevertheless, the high similarity between $\mathrm{PpS}_{9}$ and $\mathrm{PcS}_{117}$ from P. communis was already described in [16]. The occurrence of hybridization events between P. communis and other wild species is also confirmed by the high degree of homology between the $S$-RNase alleles of P. pyraster and those of P. communis [20]. Furthermore, many plants identified as P. pyraster likely 
represent various stages of hybridization between $P$. pyraster and P. communis [32]. Such close genetic proximity of the wild accessions to most of the LVs could also be explained by their wide use as rootstock to propagate selected varieties, as well as increase plant vigor and adaptability in different pedoclimatic conditions [33].

Collectively, the $S$-genotyping results confirmed the existence of genetic distinctness between the "wild" and "cultivated" subpopulations which emerged from previous SSR analyses. While natural and human selections indeed shaped the population genetic structure differently, forced allogamy and insect-mediated pollination favored gene flow between wild and cultivated populations. It is reasonable to hypothesize that at least some of the ICVs did not come in contact with the Sicilian pear populations, preventing gene exchange with local genotypes; however, those cultivars and genotypes which were introduced in Sicily in historical times offered "new" S-alleles that had the chance to spread into the local gene pool. It should be considered that, unlike other loci, the S-locus is subject to frequency-dependent balancing selection; i.e., pollen harboring rare alleles has increased chances to be accepted by pistils with respect to more frequent ones [34], making the frequency of a rare allele increase across generations until an equilibrium is reached. In such a scenario, an $S$-allele introduced in Sicily through foreign cultivars would not only rapidly spread in the local population (thanks to the ability of its pollen to be accepted by $100 \%$ of local pistils), but would then have a great chance to become a stable part of the local gene pool and to be maintained for long times in the population, as frequency-dependent balancing selection makes it very unlikely to loose $S$-alleles due to random frequency fluctuations or genetic drift [35]. Natural selection, therefore, might have favored the introgression of new $S$-alleles from cultivated to wild populations; however, on the other hand, the opposite path (from wild to cultivated material) would be theoretically more unlikely to occur, as human selection tends to eliminate wild-related detrimental traits, which in most cases affect hybrid progenies. On the basis of these assumptions, wild populations are expected to maintain a greater allelic diversity at the S-locus than cultivated ones. The SSR-based data on population structure previously described, combined with the $S$-genotypes determined in this study, support the following hypothesis: when the two groups supposed to correspond to "wild" and "cultivated" subpopulations according to SSR data are analyzed separately, the former shows a higher number of S-alleles than the latter (19 vs. 11; Figure 1). Moreover, allele frequencies in the "wild" subgroup are less skewed, with none of the alleles reaching 10\%, while, in the "cultivated" group, only two alleles accounted for more than $50 \%$ of the allelic composition of the entire subpopulation $\left(\mathrm{PcS}_{101}\right.$ and $\mathrm{PcS}_{103} ;$ Figure 1$)$. The S-allele composition of the "wild" group is, therefore, less distant from an equilibrium state, in which natural balancing selection tends to maintain comparable frequencies for all the $S$-alleles present in the population.

\section{Conclusions}

The S-allele genotyping analysis was conducted on an ex situ collection encompassing most of the local Sicilian varieties selected for their traits of agronomic interest complemented with national/international cultivars and with related wild species. Results shed light on the distribution of the $S$-alleles among accessions and revealed that RSs display a high diversity from ICVs, in terms of the S-allele composition. On the other hand, LSs and NCVs showed a more heterogeneous distribution of the $S$-alleles as the results of a more complex history of hybridization. The analysis of the $S$-allele distribution provided novel insight into the contribution of RSs and ICVs to the genetic background of the LVs and NCVs. Furthermore, these results provide information that can be readily employed by breeders for the set-up of novel mating schemes, both for rootstocks and varieties, and they are the ideal completion of the phenotypic and genotypic evaluation of the Mount Etna pear germplasm described by Ferlito et al. (under review) and Bennici et al. [17].

Supplementary Materials: The following are available online at http://www.mdpi.com/1999-4907/11/11/1228/s1: Table S1. Allele-specific primer pairs used for S-genotyping; Table S2. S-Genotypes assigned to the accessions in analysis combining consensus and allele-specific PCR primers. 
Author Contributions: Conceptualization, G.D. and L.D.; methodology: G.D. and P.D.F.; validation G.L.C. and P.D.F.; formal analysis, S.B. and M.D.G.; resources F.F.; data curation, M.D.G.; writing—original draft preparation S.B. and M.D.G.; writing-review and editing G.D., A.G., and S.L.M. All authors read and agreed to the published version of the manuscript.

Funding: This research received no external funding.

Conflicts of Interest: The authors declare no conflict of interest.

\section{References}

1. McClure, B.; Haring, V.; Ebert, P.; Anderson, M.; Simpson, R.; Sakiyama, F.; Clarke, A. Style self-incompatibility gene products of Nicotiana alata are ribonucleases. Nature 1989, 342, 955-957. [CrossRef] [PubMed]

2. De Nettancourt, D. Incompatibility and Incongruity in Wild and Cultivated Plants; Springer: Berlin, Germany, 2001; Volume 3.

3. Broothaerts, W.; Janssens, G.A.; Proost, P.; Broekaert, W.F. cDNA cloning and molecular analysis of two self-incompatibility alleles from apple. Plant Mol. Biol. 1995, 27, 499-511. [CrossRef] [PubMed]

4. Sassa, H.; Kakui, H.; Miyamoto, M.; Suzuki, Y.; Hanada, T.; Ushijima, K.; Kusaba, M.; Hirano, H.; Koba, T. S Locus F-Box Brothers: Multiple and Pollen-Specific F-Box Genes With S Haplotype-Specific Polymorphisms in Apple and Japanese Pear. Genetics 2007, 175, 1869-1881. [CrossRef] [PubMed]

5. De Franceschi, P.; Dondini, L.; Sanzol, J. Molecular bases and evolutionary dynamics of self-incompatibility in the Pyrinae (Rosaceae). J. Exp. Bot. 2012, 63, 4015-4032. [CrossRef] [PubMed]

6. Zuccherelli, S.; Tassinari, P.; Broothaerts, W.; Tartarini, S.; Dondini, L.; Sansavini, S. S-Allele characterization in self-incompatible pear (Pyrus communis L.). Sex. Plant Reprod. 2002, 15, 153-158. [CrossRef]

7. Sanzol, J.; Herrero, M.B. Identification of self-incompatibility alleles in pear cultivars (Pyrus communis L.). Euphytica 2002, 128, 325-331. [CrossRef]

8. Zisovich, A.; Stern, R.; Sapir, G.; Shafir, S.; Goldway, M. The RHV region of S-RNase in the European pear (Pyrus communis) is not required for the determination of specific pollen rejection. Sex. Plant Reprod. 2004, 17, 151-156. [CrossRef]

9. Sanzol, J.; Sutherland, B.G.; Robbins, T.P. Identification and characterization of genomic DNA sequences of the S-ribonuclease gene associated with self-incompatibility alleles S1 to S5 in European pear. Plant Breed. 2006, 125, 513-518. [CrossRef]

10. Takasaki, T.; Moriya, Y.; Okada, K.; Yamamoto, K.; Iwanami, H.; Bessho, H.; Nakanishi, T. cDNA cloning of nine $\mathrm{S}$ alleles and establishment of a PCR-RFLP system for genotyping European pear cultivars. Theor. Appl. Genet. 2006, 112, 1543-1552. [CrossRef]

11. Moriya, Y.; Yamamoto, K.; Okada, K.; Iwanami, H.; Bessho, H.; Nakanishi, T.; Takasaki, T. Development of a CAPS marker system for genotyping European pear cultivars harboring $17 \mathrm{~S}$ alleles. Plant Cell Rep. 2007, 26, 345-354. [CrossRef]

12. Sanzol, J.; Robbins, T.P. Combined Analysis of S-Alleles in European Pear by Pollinations and PCR-based S-Genotyping; Correlation between S-Phenotypes and S-RNase Genotypes. J. Am. Soc. Hortic. Sci. 2008, 133, 213-224. [CrossRef]

13. Goldway, M.; Takasaki-Yasuda, T.; Sanzol, J.; Mota, M.; Zisovich, A.; Stern, R.A.; Sansavini, S. Renumbering the S-RNase alleles of European pears (Pyrus communis L.) and cloning the S109 RNase allele. Sci. Hortic. 2009, 119, 417-422. [CrossRef]

14. Sanzol, J. Genomic characterization of self-incompatibility ribonucleases (S-RNases) in European pear cultivars and development of PCR detection for 20 alleles. Tree Genet. Genomes 2009, 5, 393-405. [CrossRef]

15. Sanzol, J. Pistil-function breakdown in a new S-allele of European pear, S21 ${ }^{\circ}$, confers self-compatibility. Plant Cell Rep. 2008, 28, 457-467. [CrossRef] [PubMed]

16. Gharehaghaji, A.N.; Arzani, K.; Abdollahi, H.; Shojaeiyan, A.; Dondini, L.; De Franceschi, P. Genomic characterization of self-incompatibility ribonucleases in the Central Asian pear germplasm and introgression of new alleles from other species of the genus Pyrus. Tree Genet. Genomes 2014, 10, 411-428. [CrossRef]

17. Bennici, S.; Casas, G.L.; Distefano, G.; Di Guardo, M.; Continella, A.; Ferlito, F.; Gentile, A.; La Malfa, S. Elucidating the contribution of wild related species on autochthonous pear germplasm: A case study from Mount Etna. PLoS ONE 2018, 13, e0198512. [CrossRef] 
18. Matsumoto, K.; Tamura, F.; Chun, J.-P.; Tanabe, K. Native Mediterranean Pyrus Rootstock, P. amygdaliformis and P. elaeagrifolia, Present Higher Tolerance to Salinity Stress Compared with Asian Natives. J. Jpn. Soc. Hortic. Sci. 2006, 75, 450-457. [CrossRef]

19. Łukasz, W.; Antkowiak, W.; Lenartowicz, E.; Bocianowski, J. Genetic diversity of European pear cultivars (Pyrus communis L.) and wild pear (Pyrus pyraster (L.) Burgsd.) inferred from microsatellite markers analysis. Genet. Resour. Crop. Evol. 2010, 57, 801-806. [CrossRef]

20. Łukasz, W.; Antkowiak, W.; Sips, M.; Slomski, R. Self-incompatibility alleles in Polish wild pear (Pyrus pyraster (L.) Burgsd.): A preliminary analysis. J. Appl. Genet. 2010, 51, 33-35. [CrossRef]

21. Ye, J.; Coulouris, G.; Zaretskaya, I.; Cutcutache, I.; Rozen, S.G.; Madden, T.L. Primer-BLAST: A tool to design target-specific primers for polymerase chain reaction. BMC Bioinform. 2012, 13, 134. [CrossRef]

22. R Core Team. R: A Language and Environment for Statistical Computing; R Foundation for Statistical Computing: Vienna, Austria, 2017.

23. Tassinari, P. Analisi molecolari ed agronomiche dell'incompatibilita' gemetofitica in pero (Pyrus communis) e ciliegio (Prunus avium e P. cerasus): Ricerche sul locus S. Ph.D. Thesis, University of Bologna, Bologna, Italy, 2005.

24. Mota, M.; Tavares, L.; Oliveira, C.M. Identification of S-alleles in pear (Pyrus communis L.) cv. 'Rocha' and other European cultivars. Sci. Hortic. 2007, 113, 13-19. [CrossRef]

25. Takasaki-Yasuda, T.; Nomura, N.; Moriya-Tanaka, Y.; Okada, K.; Iwanami, H.; Bessho, H. Cloning anS-RNaseallele, including the longest intron, from cultivars of European pear (Pyrus communis L.). J. Hortic. Sci. Biotechnol. 2013, 88, 427-432. [CrossRef]

26. Rehder, A. A Manual of Cultivated Trees and Shrubs Hardy in North American Exclusive of the Subtropical and Warmer Temperate Regions, 2nd ed.; Macmillan: New York, NY, USA, 1940; p. 403.

27. Katayama, H.; Tachibana, M.; Iketani, H.; Zhang, S.-L.; Uematsu, C. Phylogenetic utility of structural alterations found in the chloroplast genome of pear: Hypervariable regions in a highly conserved genome. Tree Genet. Genomes 2011, 8, 313-326. [CrossRef]

28. Volk, G.M.; Henk, A.D.; Richards, C.M.; Bassil, N.; Postman, J. Chloroplast sequence data differentiate Maleae, and specifically Pyrus, species in the USDA-ARS National Plant Germplasm System. Genet. Resour. Crop. Evol. 2018, 66, 5-15. [CrossRef]

29. Liu, Q.; Song, Y.; Liu, L.; Zhang, M.; Sun, J.; Zhang, S.; Wu, J. Genetic diversity and population structure of pear (Pyrus spp.) collections revealed by a set of core genome-wide SSR markers. Tree Genet. Genomes 2015, 11, 128. [CrossRef]

30. Teng, Y.; Tanabe, K.; Tamura, F.; Itai, A. Genetic Relationships of Pyrus Species and Cultivars Native to East Asia Revealed by Randomly Amplified Polymorphic DNA Markers. J. Am. Soc. Hortic. Sci. 2002, 127, 262-270. [CrossRef]

31. Kumar, S.; Kirk, C.; Deng, C.; Wiedow, C.; Knaebel, M.; Brewer, L. Genotyping-by-sequencing of pear (Pyrus spp.) accessions unravels novel patterns of genetic diversity and selection footprints. Hortic. Res. 2017, 4, 1-10. [CrossRef]

32. Dolatowski, J.; Podyma, W.; Szymañska, M.; Zych, M. Molecular studies on the variability of Polish semi-wild pears (Pyrus) using AFLP. J. Fruit Ornam. Plant Res. 2004, 12, 331-337.

33. Volk, G.M.; Cornille, A. Genetic Diversity and Domestication History in Pyrus. In The Pear Genome; Korban, S.S., Ed.; Springer: Cham, Germany, 2019; pp. 51-62.

34. Wright, S. The Distribution of Self-Incompatibility Alleles in Populations. Evolution 1964, 18, 609. [CrossRef]

35. Ioerger, T.R.; Clark, A.G.; Kao, T.H. Polymorphism at the self-incompatibility locus in Solanaceae predates speciation. Proc. Natl. Acad. Sci. USA 1990, 87, 9732-9735. [CrossRef]

Publisher's Note: MDPI stays neutral with regard to jurisdictional claims in published maps and institutional affiliations. 